\title{
Reconstruction - Emotion - Narrative Discussion on the Application of Cinematic Space Language in Interior Design
}

\author{
Wen Lan ${ }^{1, *}$ \\ ${ }^{1}$ Xiamen Academy of Arts and Design, Fuzhou University, Xiamen, Fujian, China \\ *Corresponding author. Email: 892710043@qq.com
}

\begin{abstract}
The article discusses and experiments on the formal language and design methods of film intervention in interior space design. It explores how emotion and narrative can be organically combined with cinematic techniques, and how to construct the place spirit of interior space based on film montage technique. The organic combination of these two art forms can enrich the diversity of interior design and provide more possibilities for future development.
\end{abstract}

Keywords: space, film, montage, narrative and emotion

\section{INTRODUCTION}

Since the development of media art, the correlation between film and architectural space has been one of the themes discussed by relevant architects. In order to make space design more creative and narrative, and to create a strong or peaceful multi-dimensional emotional level, which can enrich the experience function of space, the designer integrates the cinematic space language into the construction activities of the real space environment. It can better convey the place spirit of the space and give the space soul and vitality through the cross-border secondary creation.

\section{FILM AND SPACE}

\section{A. The space of film}

In contemporary society, the film occupies an important part of human social life with its inclusive artistic expression. A film needs to be based on the creator's solid worldview, through various technical means to organize language, arrange characters, and form events to convey their ideas. To a certain extent, it is not only the presentation of images, but also the "space-time dialogue" behind the story.

When the viewer is watching a film, the film creates a two-dimensional image through the lens to bring the narrative organization and characters of the multidimensional space to the viewer. The film breaks the two-dimensional and three-dimensional spatial boundaries that humans can perceive, Derive a virtual space scene. At the same time, as an artistic expression form, film has more subjective attributes in controlling the dimensions, and it can meet the establishment and needs of modern people's self-space.

\section{B. Film and space}

Both film and architecture are art forms expressed by time and space, and they have a common logical basis, which is reflected in the organization of movement, narrative, and psychological organization of space and time. There are two key elements which are people and space. People in the film and architecture are connected to the space through movements and events. In real life, there are two kinds of spaces that people can perceive, one is physical space that is visible to the naked eye, and the other is virtual space that exists abstractly. If we understand that space is only a field surrounded by four sides, its value was superficial. To truly understand a space, it is necessary to experience it. The creator establishes the narrative structure through the rich composition method of space and generates the connection between time and space to convey the emotion to the viewer. Pascal Schöning said in his article "Manifesto for a Cinematic Architecture": " To achieve true spatiality, people need a black hole logic, which is so concentrated and multi-dimensional that its abundant power consumes itself to provide an incredible spatial dimension." [1] ("Fig. 1") 


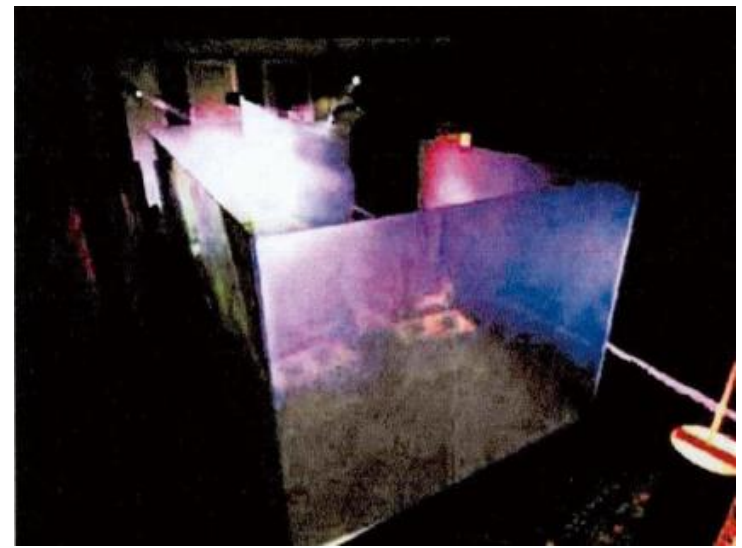

Fig. 1. Pascal Schöning: "Film House".

a. Image Source: http://image.baidu.com

In the context of contemporary design, postmodernism pursues people-oriented thinking even more. As a self-independent individual, people are no longer satisfied only with " necessity ", which is the traditional material needs, but to pursue spiritual selfsatisfaction. Space design pays more attention to people's feelings and experiences, which coincides with the movie. The Cinematic architecture in modern theory is to try to create a building space and perceptual model that pay attention to people's psychological feelings and emotional needs through the research and study of film art expression techniques. [2]

Through case design analysis, this article uses the montage technique to establish the virtual self in space, study the relationship between film and space and how to build a cinematic space language structure, and discuss the formal language of film intervening in indoor space and how to establish the place spirit of indoor space.

\section{THE CINEMATIC LANGUAGE OF SPACE}

\section{A. Film translation}

As mentioned above, the film organizes the spacetime language based on the creator's subjective consciousness. It conveys a rich virtual space experience to the viewer through the lens. To achieve the purpose of converting the content perceived by the viewer into spatial language, the designer needs to objectively translate and subjectively interpret the film.

Because the emotional expression of the film is multi-level and multi-faceted, the film material, as an intermediary of space design, will have a big gap from the final space imaging. There is a saying in China: the whole world can be seen from a flower, and a single leaf can represent the entire linden tree. Each different interpretation direction will produce different possibilities and results. [3] In this way, the multiple construction of space can be greatly enriched, and unpredictability will create surprises. In this process, designers need to observe and think about how to use their own language to reproduce the events in the movie, not only pay attention to the organization of narrative line, but also pay attention to the capture and expression of emotions. Because pure narrative is easy to float on the surface, the construction of spatiality also needs thematic emotions. It is necessary to grasp the construction point of consciousness and make it materialized into a space form, so as to enrich the viewer's knowledge and understanding of space.

\section{B. Image reconstruction}

After we systematically complete the translation of the movie, we can reconstruct the image space through various forms of montage. Montage was originally a term for French architecture, meaning composition and assembly. Later, it was developed in the art of video and formed a rather powerful lens combination theory. The montage of the movie creates complex moving lines in the movie lens by breaking up, reorganizing, splicing, etc. It combines some unrelated pictures together to produce a metaphorical formal language, artificially create expressions and experiences of subjective emotions. As a spatial combination method, montage can well structure the subjective world and objective existence, thereby transforming local elements into a general methodology, and convey the creator's thoughts and emotions to the viewer.

\section{Emotional expression}

Montages are mainly divided into two types in film studies. One is narrative montage, which has parallel montages, cross montages, repeated montages, and continuous montages. The other is performance montage, which include metaphor montages, contrast montages, psychological montages, and accumulation Montage, etc. These methods are commonly used in behavioral activities that use structural techniques to convey emotions. The designer thinks about how to use these forms to accurately translate the film and form a spatial language throughout the design process. The object is not only a single spatial scene but also a combination of multiple spaces, sorting and integration, using various media to grasp the theme Create space. The common feature of film and space is to express emotions through events. The application of montage must be based on the integration and expression of emotions. In the order of space organization and image construction, it emphasizes the main role of people in space and the perception of people in spirit. 


\section{THE CINEMATIC TECHNIQUE OF SPACE AND ITS APPLICATION}

\section{A. Film themes and keywords}

The following takes "E - I Motion Concept Space Design" as an example to analyze how to apply the cinematic design method of space. The theme word "E . I" originated from the 2014 science-fiction movie "Interstellar" directed by American director Christopher Nolan. ("Fig. 2") The film tells the story of a father who was sent to space to find a new home for mankind and found a way to save mankind through love with his daughter when human civilization is at stake. After a comprehensive analysis of the film, the first extracted content is the keywords that capture the viewer's mood: occurrence, encounter, love and hope. Therefore, the subject words can be interpreted as follows: "E" stands for emotion, and "I" stands for time, and the entire theme can also be translated as only love can travel through time and space.

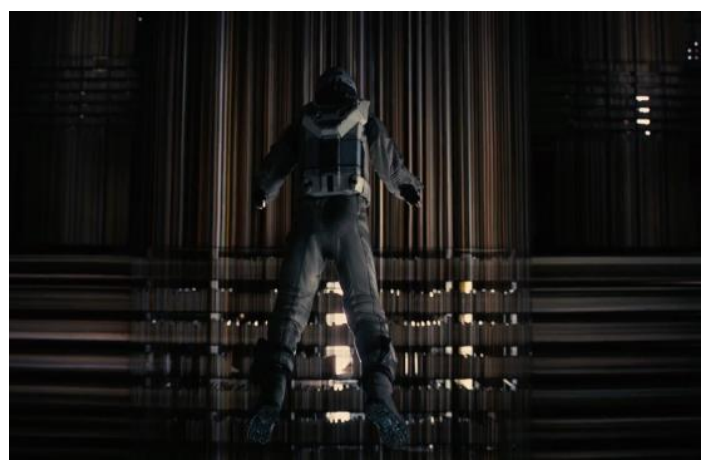

Fig. 2. The male character of "Interstellar" entered the wormhole.

Image Source: http://image.baidu.com

\section{B. Narrative structure and spatial organization}

The narrative is a literary term. It is precisely because of the occurrence of events that the space has an emotional level, which in turn generates a place spirit. In the design process, the connection between the film narrative and the project space becomes very important. The plot of the film can be divided into two story lines, one happening on the earth and the other happening in outer space. Under two parallel timelines, events continue to occur to promote the plot of the film. The creators use parallel montages to intersperse the story development of the two timelines. The project address is an underground city in a Beijing shopping mall. There are two floors of underground space, so we can import the film to sort out and define the space: the space on the upper and lower floors is also a parallel process, and the negative one is set as the earth story structure line, the negative second floor is set as the space story structure line, and the two are connected by a wormhole in the middle, that is, the stairs. ("Fig. 3")
Viewers can enter from the negative first floor or from the negative second floor. In terms of space layout, sports space with a small footprint such as fitness equipment, gymnasiums, and baths, are located on the negative first floor, while sports spaces with a large footprint, such as basketball courts and swimming pools, are located on the negative second floor. This is not to limit the spatial experience through a fixed order, but to lock the spatial emotion through open spatial focusing. Each space has a corresponding source of film structure material, and the spatial relationship can be organized according to the story logical relationship established by the movie creator.

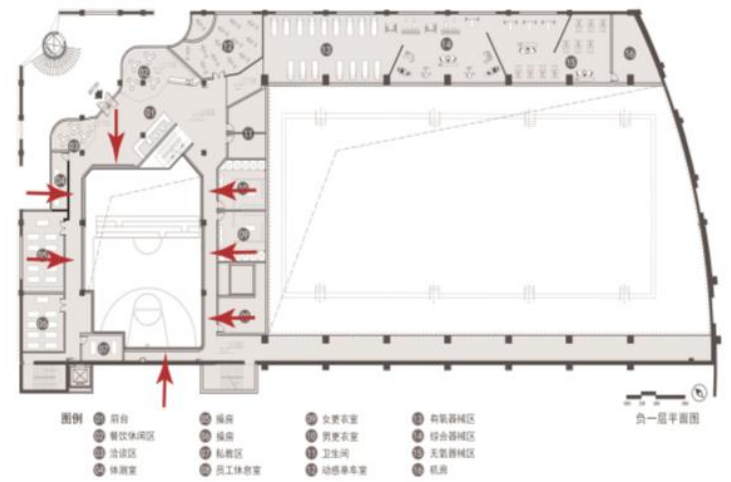

Fig. 3. Negative floor plan and viewpoint of "Murphy's Bookshelf" image window.

Image Source::drawn by the autho

\section{Lens language and spatial construction}

Based on the narrative structure and spatial organization of the film, the designer sorts out the picture composition of the film, arranges the overall color of the space reasonably, the spatial shape materialized according to the internal logic, and the material expression space combined with the material, which is the whole of the filmed lens language "landing" space process.

The entrance of the space should shape the character of the space, let the viewer grasp the emotion of the space, and establish the "first impression". Therefore, the entrance space on the negative level is divided into two spatial compositions by the aisle, the stable order on one side expresses the static state, and the tilt on the other side irregularly expresses the moving state. By application of contrast montage, the penetration of two opposite emotional states in the space guides the viewer into the sports space of different attributes. The arrangement of these sports spaces is also determined according to the difference in movement status. The entrance of the negative second floor is first covered by the wall, which is also a process of transitional emotions and filtering visual psychological feelings, so 
as to achieve the purpose of enhancing the viewer's visual perception after entering the space.

The visual intersection of the entrance of the negative first floor and the basketball court of the negative second floor come from the creator's shot of Murphy's bookshelf and the multidimensional space in the wormhole in the film "Interstellar". In the development of the plot of the film, Murphy's daughter's bookshelf is undoubtedly a vital clue to the whole story, and it is a key design element. The father found a way to convey the message through the bookshelf in the daughter's room and a watch on the shelf in the wormhole and successfully transmitted his discovery about how to save humanity. The bookshelf connecting the two spaces acts like a wormhole in the film or a window in the actual project space. In the design of this scheme, "Murphy's Bookshelf" is actually a block-type window device, by using colored transparent glass in the form of imitation bookshelves to combine multiple floor-to-ceiling "image" Windows, The viewers on the first and second floors communicate visually from multiple perspectives, just like the father in the film saw images from different time and space through the bookshelf. And this kind of communication has undergone secondary conduction due to the characteristics of the window material, namely the colored glass, it is not directly presented to the viewer. According to the different rotation angles of the components, the images presented by them are also different: when the rotation angle is $0^{\circ}$, the two spaces are isolated by the components, although it is still visible, it seems to be a virtual representation of different dimensions; When it is equal to $0^{\circ}$, there will be an intersection between the spaces, ("Fig. 4") and the viewers on the upper and lower floors have different degrees of communication, which strengthens the atmosphere and fun of the space. ("Fig. 5")

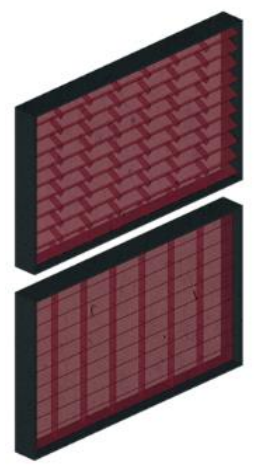

Fig. 4. "Murphy's Bookshelf" Video Window Components.

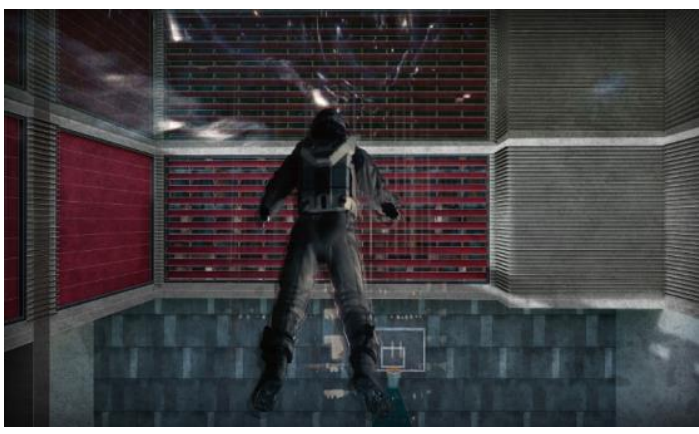

Fig. 5. "Murphy's Bookshelf" Video Window.

Similarly, the space is divided into two video story lines, and the staircase is also a turning point for communicating two parallel spaces, just like a wormhole. Therefore, in the staircase space, the space image that passed by the black hole when the father expedition team entered the solar system was taken as the source of the design material, and the infinitely circular circle is used as the design element to guide the space. The circle is actually a typical representative of the infinite cycle. In this space, all objects are infinitely extended, changed and circulated, and there is no clear spatial boundary. On the basis of extracting the composition of the image, the mirror is used as the main material of the wall, and the function of using the mirror to refract and reflect objects creates a space atmosphere, which plays a role in causing viewers to change their mood and introduce space.

From the stairs to the negative second floor, there are mainly two spaces: the basketball court and the swimming pool. The corresponding movie plot is the scene experienced by the wormhole and the hero's exploration on other planets. Continue to use the keywords and elements of the space design, the basketball court encloses the space through continuous wall modular components and mirror material ceilings. In addition to the composition that satisfies the functional requirements of space, other concerns are the perception of viewers in space, and perception produces emotion. A metaphoric montage is used here. The infinite continuation and expansion of the wormhole space implies a variety of possibilities. As Murphy's Law in the film says: "What may happen will always happen, and people can only grasp the present."

The swimming pool is the largest physical area in the entire sports space. The continuous regular components and the infinitely extending mirror ceiling only occupy a part of the space design. The design uses a contrast montage technique, inspired by the composition of the images in the film. In such a space, the viewer is just a tiny part, just as humans exist in the universe like ants. The space creates a space atmosphere through large areas of blank walls, 
continuous components, small areas of doors and windows, and moving water bodies. Different texture materials and different areas of space constitute a strong contrast, and the contrast mobilizes viewers' emotions.

In the process of image construction, the acquisition and establishment of space colors are not only dependent on the screen, but also set according to the narrative theme of the film. Black represents unknown, red represents emotion, green represents hope, and yellow represents warning. Different color combinations represent different narrative structures, and the viewer's perceptions caused by them have rich possibilities.

\section{CONClusion}

The cinematic space design focuses on emotions, narratives, and the spiritual feelings brought by the space. Its internal logic has a tangible impact on the development of modern design concepts. The close connection between space and film is accidental and inevitable. Although the requirements for architectural space have changed in different times, the constant is that the impact of human beings as the subject of space on the space, the importance of human emotions in space design will never change. Studying the combination of various art forms and space design can provide more possibilities for the future.

\section{References}

[1] Pascal Schöning, Dou Pingping, "Manifesto for a Cinematic Architecture," Architect, June 2008, pp.81-86.

[2] Wang Mengjie, "Preliminary Research on the Spiritual Construction of Places in the Old City Reconstruction Based on Film Architecture," Changchun Institute of Technology, December 2008, pp.20-21.

[3] Liu Si, "The Cross-Border Text-Translation and Translation of Film and Architecture," New Architecture, December 2008 , pp.62-64.

[4] Yang Chen, "Film Architecture-A New Exploration of the Expression of Architectural Art," Central Academy of Fine Arts, March 2013 , pp.37-39.

[5] Zhang Yonghe, "The Game from the Back Window to the View," Architecture and Culture, February 1994, pp.34-37.

[6] Li Menghai. Niu Shengnan. Ning Shao, "Narrative Space Construction Based on Film Montage," Architecture and Culture, February 2019, pp.172-174.

[7] Sun Chuyi, "Exploring the Architectural Language of Imaging - Taking Space Montage as an Example," Architecture and Culture, March 2019, pp.177-178. 